\title{
Um framework para adaptação automática de jogos sérios considerando as emoções e os traços de personalidade do jogador
}

\author{
Renan Vinicius Aranha \\ renanvinicius@usp.br \\ Escola Politécnica \\ Universidade de São Paulo
}

\author{
Fátima de Lourdes dos Santos Nunes \\ fatima.nunes@usp.br \\ Escola de Artes, Ciências e Humanidades \\ Universidade de São Paulo
}

\section{RESUMO}

A utilização de jogos sérios em diversos contextos visa a tornar uma atividade ou processo mais atrativo para o usuário. Neste contexto, o envolvimento do jogador apresenta-se como um fator de grande importância para o sucesso da aplicação computacional. Tal característica propicia um cenário desafiador, em que a utilização de técnicas de Computação Afetiva pode apresentar inúmeros benefícios ao possibilitar a adaptação automática do jogo conforme o estado emocional do usuário. A literatura científica apresente diversos alguns estudos neste sentido, no entanto, a utilização destas informações pode ser eficaz para identificar quando se deve executar uma adaptação no jogo, mas pode ser insuficiente para definir uma adaptação que atenda as necessidades individuais de cada usuário. Com o intuito de otimizar este processo, esta pesquisa propõe o aprimoramento de um framework de Computação Afetiva ao incluir a identificação dos traços de personalidade do usuário com o intuito de oferecer adaptações que não consideram apenas o estado emocional, mas também as características de cada indivíduo.

\section{CCS CONCEPTS}

- Human-centered computing $\rightarrow$ User models; HCI theory, concepts and models.

\section{KEYWORDS}

Computação Afetiva, Traços de Personalidade, Jogos sérios

Permission to make digital or hard copies of all or part of this work for personal or classroom use is granted without fee provided that copies are not made or distributed for profit or commercial advantage and that copies bear this notice and the full citation on the first page. Copyrights for components of this work owned by others than ACM must be honored. Abstracting with credit is permitted. To copy otherwise, or republish, to post on servers or to redistribute to lists, requires prior specific permission and/or a fee. Request permissions from permissions@acm.org.

IHC 2019, Outubro 21-25, 2019, Vitória, ES

(C) 2019 Association for Computing Machinery.

ACM ISBN 978-1-4503-9999-9/18/06_..\$15.00
ACM Reference Format:

Renan Vinicius Aranha and Fátima de Lourdes dos Santos Nunes. 2019. Um framework para adaptação automática de jogos sérios considerando as emoções e os traços de personalidade do jogador. In XVIII Simpósio Brasileiro sobre Fatores Humanos em Sistemas Computacionais.

\section{INTRODUÇÃO}

Jogos sérios têm sido utilizados em diversos cenários com o intuito de tornar mais atrativos para os usuários processos e atividades que, tradicionalmente, são considerados enfadonhos $[6,7,10]$. Neste tipo de jogo, a manutenção do envolvimento do usuário apresenta-se como um fator de grande importância para o sucesso da aplicação computacional. Por esta razão, pesquisadores têm investigado estratégias que possam contribuir com a manutenção do envolvimento - ou do "estado de flow" do usuário - durante a utilização de um software $[2,9]$. O termo "estado de flow" é utilizado para definir o estado em que o usuário sente-se plenamente envolvido em uma atividade agradável, que lhe proporciona grande satisfação [4].

Dentre as diversas estratégias que visam a manutenção do estado de flow, destaca-se a adaptação automática de aplicações computacionais a partir do estado emocional do usuário, chamada de "adaptação afetiva" [18]. Nesta abordagem, que utiliza técnicas de Computação Afetiva (CA), o estado emocional do usuário é reconhecido e uma adaptação é realizada no software em tempo real, de acordo com a emoção identificada.

Uma revisão sistemática de literatura acerca da utilização de técnicas de Computação Afetiva para a adaptação automática de software aponta que, usualmente, as aplicações de adaptação afetiva não consideram características únicas dos indivíduos - além do estado emocional - para a escolha da adaptação afetiva [2]. Em contraponto, Ortigosa et al. [12] afirmam que "a modelagem do usuário é essencial no contexto de sistemas adaptativos”, sendo a identificação da personalidade do usuário importante para que suas necessidades potenciais em diferentes contextos sejam conhecidas. Ortigosa et al. [12] reforçam ainda que "as aplicações adaptativas podem se beneficiar de ter modelos de personalidade 


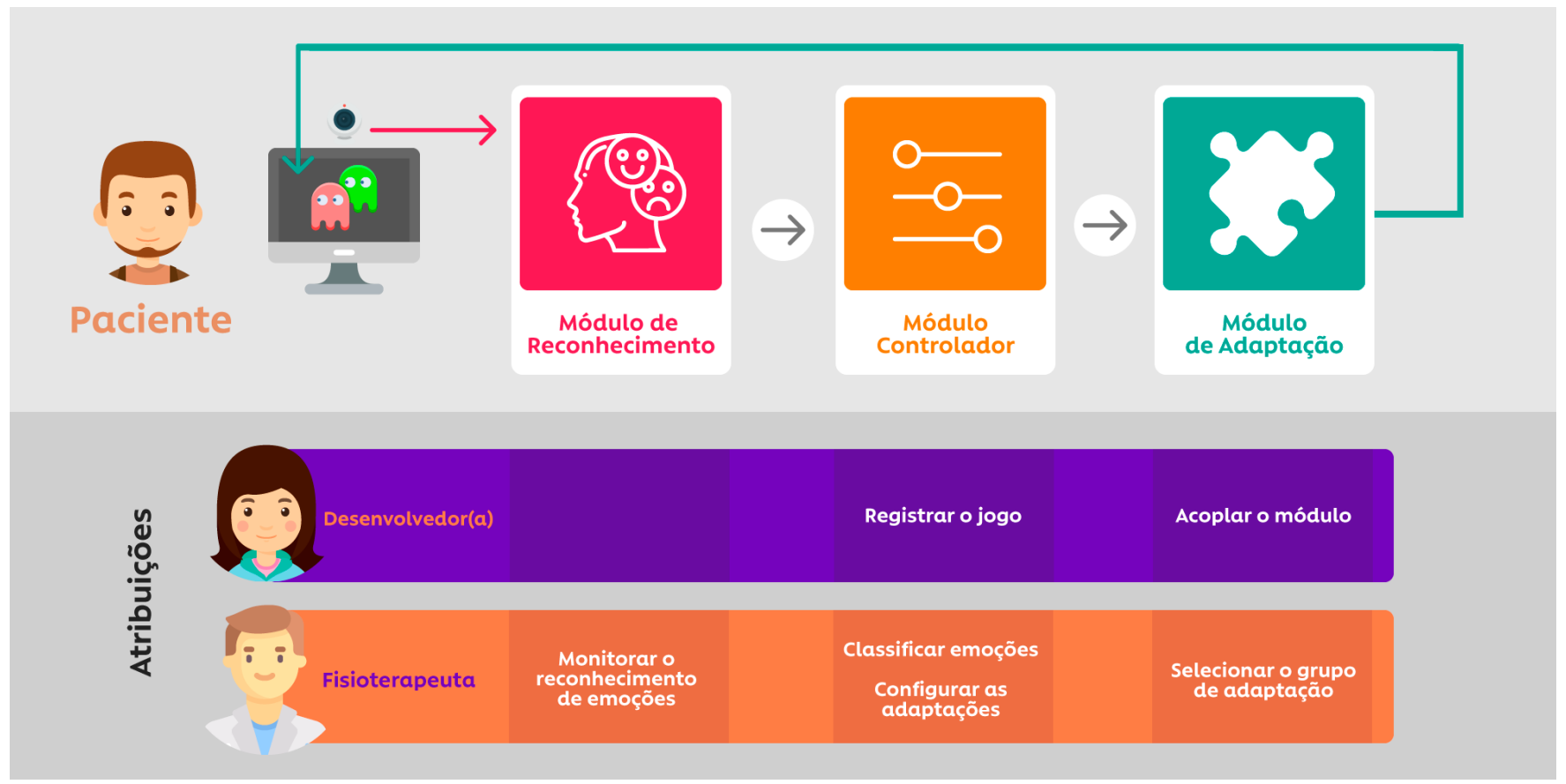

Figura 1: Composição do framework EasyAffecta, proposto por Aranha et al. [3] (adaptado), com a atribuição de cada usuário nos módulos que compõem o framework.

dos usuários para adaptar seu comportamento de acordo com a personalidade de cada usuário”.

O framework EasyAffecta, proposto por Aranha et al. [3], prevê a adaptação afetiva de jogos sérios para reabilitação motora com personalização para cada paciente. Todavia, coube ao fisioterapeuta realizar a análise das preferências do usuário. Embora esta abordagem tenha apresentado resultados positivos, a classificação manual dos usuários torna-se inviável em várias situações, como a existência de um grande número de usuários ou quando a aplicação não disponibiliza interfaces apropriadas para configuração.

Uma das abordagens que pode contribuir com este cenário consiste na análise dos traços de personalidade dos usuários para a recomendação das adaptações afetivas que serão efetuadas no jogo. A Teoria dos Traços de Personalidade é um campo de estudo da Psicologia que busca representar a personalidade humana por meio de traços - como, por exemplo, extroversão e abertura a experiências. Tais informações acerca do usuário podem ser obtidas por meio da utilização de questionários ou de técnicas computacionais [17].

A análise dos traços de personalidade pode ser uma importante ferramenta de apoio para a definição das adaptações afetivas, possibilitando uma melhor compreensão sobre os estados emocionais do usuário e os elementos que podem propiciar maior engajamento.
A literatura científica apresenta trabalhos que analisam dados do usuário para diferentes propósitos, como a análise de sentimento, $[1,11,14]$, o reconhecimento dos traços de personalidade $[5,13]$, além da adaptação de software e a recomendação de conteúdo $[8,15,16]$. Todavia, a utilização dos traços de personalidade em conjunto com o estado emocional do usuário tem sido uma abordagem pouco explorada na literatura.

Portanto, esta pesquisa tem como objetivo geral propor e implementar uma abordagem para propiciar adaptação automática de jogos sérios usando Computação Afetiva e traços de personalidade. Além da abordagem conceitual, métodos e técnicas serão desenvolvidos para compor um framework que viabilizará o desenvolvimento de jogos sérios utilizando esta abordagem. Embora a abordagem possa ser utilizada em qualquer tipo de aplicação, a prova de conceito no contexto desta pesquisa se dará no contexto de jogos sérios, considerandose a importância da manutenção do engajamento do usuário neste tipo de aplicação.

\section{DEFINIÇÃO DA PROPOSTA}

Como prova de conceitos, o framework Easy Affecta, proposto por Aranha et al. [3], será aprimorado para possibilitar a definição de adaptações afetivas conforme os traços de personalidade do usuário. A Figura 1 ilustra a arquitetura 
Um framework para adaptação automática de jogos

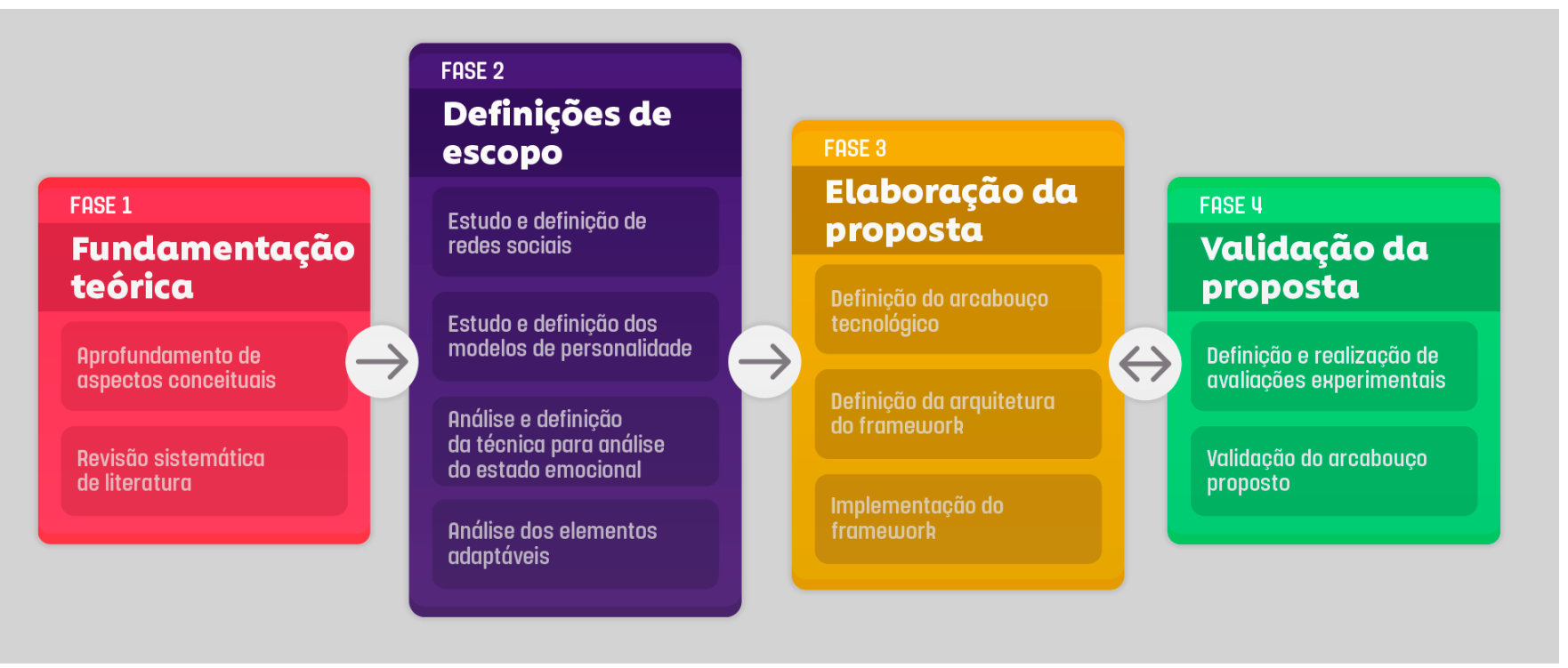

Figura 2: Fases da metodologia de desenvolvimento da pesquisa.

da ferramenta, composta por: (a) um módulo de Reconhecimento, responsável por capturar sinais de entrada do usuário e inferir o estado emocional do mesmo; (b) um módulo denominado Controlador Emoção - Ação, responsável por executar uma ação na aplicação, em resposta à emoção identificada pelo módulo anterior e (c) um módulo de Adaptação, inserido na aplicação final, que recebe as instruções a serem executadas.

Embora esta abordagem tenha apresentado resultados positivos, entende-se que seu impacto pode ser otimizado ao automatizar o processo de classificação do perfil do usuário. Conforme discutido anteriormente, no EasyAffecta tal atividade é realizada manualmente pelo fisioterapeuta, que até então precisa conhecer as preferências do jogador para configurar as adaptações adequadamente. Neste cenário, a utilização de instrumentos ou modelos que possibilitem a análise de traços do usuário pode apresentar inúmeros benefícios.

\section{MÉTODOS}

Visando-se ao atendimento dos objetivos propostos, a condução deste projeto de pesquisa se dividirá em blocos de tarefas (ilustrados pela Figura 2), a saber:

\section{Fundamentação teórica}

Visando ao aprimoramento dos aspectos conceituais, além de pesquisas exploratórias, foram realizadas duas revisões sistemáticas de literatura: i) uma análise acerca dos impactos dos traços de personalidade na experiência do usuário em sistemas de Realidade Virtual; ii) uma investigação sobre a utilização dos traços de personalidade para a realização de adaptação em software.

\section{Definições de escopo}

Este estágio compreende o estudo e a definição da abordagem de coleta de dados do usuário, bem como da identificação dos traços de personalidade do mesmo. Ainda neste escopo inclui-se, a partir de uma revisão sistemática de literatura, a definição da técnica a ser utilizada para o reconhecimento do estado emocional do usuário e a definição dos elementos de jogabilidade a serem adaptados nos jogos sérios.

\section{Desenvolvimento da estratégia de adaptação}

A partir das definições de escopo previamente apresentadas, esta fase compreende a definição das tecnologias a serem consideradas na implementação da prova de conceitos, como também a definição e a implementação da arquitetura que irá compor a nova versão do framework EaysAffecta. Cabe destacar, ainda, que este estágio compreende a análise e definição de técnicas para a definição do elemento que será adaptado no software considerando os traços de personalidade e o estado emocional do usuário. A partir das técnicas identificadas na revisão de literatura, prevê-se a realização de diferentes avaliações experimentais.

\section{Validação da proposta}

Considerando-se os diferentes estágios de desenvolvimento desta pesquisa, prevê-se a realização de diversas avaliações experimentais, que visam desde a análise de instrumentos para a identificação dos traços de personalidade do usuário, 
bem como a identificação do impacto da abordagem proposta na manutenção do nível de engajamento do usuário.

\section{CONTRIBUIÇÕES ESPERADAS}

Em decorrência do desenvolvimento desta pesquisa de doutorado, prevê-se contribuições para as áreas de Interação Humano-Computador e Computação Afetiva, almejando especificamente os seguintes itens como resultados: i) a definição de técnicas para a identificação dos traços de personalidade do jogador; ii) o desenvolvimento e disponibilização de um framework que possibilite a adaptação de jogos sérios considerando-se o estado emocional do usuário e seus traços de personalidade; iii) avaliações com o intuito de verificar quais elementos adaptados nos jogos propiciaram maior impacto no nível de engajamento do usuário.

De modo complementar, prevê-se que o projeto tem potencial para propiciar os seguintes impactos: i) social, pois pode contribuir para melhorar o aprendizado mediado por jogos sérios; ii) tecnológico, dado que será disponibilizado um framework para possibilitar, sem grande esforço, a adaptação de jogos sérios a partir de técnicas de Computação Afetiva e dos traços de personalidade do usuário; e iii) econômico, uma vez que o arcabouço tecnológico disponibilizado contribuirá para a implementação de aplicações com maior produtividade.

\section{AGRADECIMENTOS}

O presente trabalho foi realizado com apoio da Coordenação de Aperfeiçoamento de Pessoal de Nível Superior - Brasil (CAPES) - Código de Financiamento 001.

\section{REFERÊNCIAS}

[1] Sanjida Akter and Muhammad Tareq Aziz. 2016. Sentiment analysis on Facebook group using lexicon based approach. In Electrical Engineering and Information Communication Technology (ICEEICT), 2016 3rd International Conference on. IEEE, 1-4.

[2] R. V. Aranha, C. G. Corrêa, and F. L. S. Nunes. 2019. Adapting software with Affective Computing: a systematic review. IEEE Transactions on Affective Computing (2019), 1-1. https://doi.org/10.1109/TAFFC.2019. 2902379

[3] Renan V Aranha, Leonardo S Silva, Marcos L Chaim, and Fátima LS Nunes. 2017. Using Affective Computing to automatically adapt serious games for rehabilitation. In 30th International Symposium on ComputerBased Medical Systems. IEEE.

[4] Mihaly Csikszentmihalyi. 1990. FLOW: The Psychology of Optimal Experience. Harper and Row (1990).

[5] Vitor Garcia dos Santos, Ivandré Paraboni, and Barbara Barbosa Claudino Silva. 2017. Big Five Personality Recognition from Multiple Text Genres. In International Conference on Text, Speech, and Dialogue. Springer, 29-37.

[6] Laura Freina and Michela Ott. 2015. A literature review on immersive virtual reality in education: state of the art and perspectives. In The International Scientific Conference eLearning and Software for Education, Vol. 1. "Carol I"National Defence University, 133.
[7] Cristiane M Gebara, Tito P de Barros-Neto, Leticia Gertsenchtein, and Francisco Lotufo-Neto. 2016. Virtual reality exposure using threedimensional images for the treatment of social phobia. Revista Brasileira de Psiquiatria 38, 1 (2016), 24-29.

[8] Rahul Katarya and Om Prakash Verma. 2016. Recent developments in affective recommender systems. Physica A: Statistical Mechanics and its Applications 461 (2016), 182-190.

[9] Carlos Alberto Lara, Juan Flores, Hugo Mitre-Hernandez, and Humberto Perez. 2018. Induction of Emotional States in Educational Video Games through a Fuzzy Control System. IEEE Transactions on Affective Computing (2018), 1-1. https://doi.org/10.1109/taffc.2018.2840988

[10] Mindy F Levin, Patrice L Weiss, and Emily A Keshner. 2015. Emergence of virtual reality as a tool for upper limb rehabilitation: incorporation of motor control and motor learning principles. Physical therapy 95, 3 (2015), 415-425.

[11] Preslav Nakov, Alan Ritter, Sara Rosenthal, Fabrizio Sebastiani, and Veselin Stoyanov. 2016. SemEval-2016 task 4: Sentiment analysis in Twitter. Proceedings of SemEval (2016), 1-18.

[12] Alvaro Ortigosa, Rosa M Carro, and José Ignacio Quiroga. 2014. Predicting user personality by mining social interactions in Facebook. Journal of computer and System Sciences 80, 1 (2014), 57-71.

[13] B. Y. Pratama and R. Sarno. 2015. Personality classification based on Twitter text using Naive Bayes, KNN and SVM. In 2015 International Conference on Data and Software Engineering (ICoDSE). 170-174. https: //doi.org/10.1109/ICODSE.2015.7436992

[14] Ramon Gouveia Rodrigues, Rafael Marques das Dores, Celso G CamiloJunior, and Thierson Couto Rosa. 2016. SentiHealth-Cancer: A sentiment analysis tool to help detecting mood of patients in online social networks. International journal of medical informatics 85, 1 (2016), 80-95.

[15] Olga C Santos, Jesus G Boticario, and Ángeles Manjarrés-Riesco. 2014. An approach for an affective educational recommendation model. In Recommender Systems for Technology Enhanced Learning. Springer, 123-143.

[16] Olga C Santos, Mar Saneiro, Jesus G Boticario, and MC RodriguezSanchez. 2016. Toward interactive context-aware affective educational recommendations in computer-assisted language learning. New Review of Hypermedia and Multimedia 22, 1-2 (2016), 27-57.

[17] A. Vinciarelli and G. Mohammadi. 2014. A Survey of Personality Computing. IEEE Transactions on Affective Computing 5, 3 (July 2014), 273-291. https://doi.org/10.1109/TAFFC.2014.2330816

[18] Timothy D. Wilson and Daniel T. Gilbert. 2008. Explaining Away: A Model of Affective Adaptation. Perspectives on Psychological Science 3, 5 (2008), 370-386. https://doi.org/10.1111/j.1745-6924.2008.00085.x arXiv:https://doi.org/10.1111/j.1745-6924.2008.00085.x PMID: 26158955. 